\title{
Personalizing vulvar cancer workflow in COVID-19 era: a proposal from Vul.Can MDT
}

\author{
Giorgia Garganese ${ }^{1,2} \cdot$ Luca Tagliaferri $^{3} \cdot$ Simona Maria Fragomeni $^{2}\left(\mathbb{D} \cdot\right.$ Valentina Lancellotta $^{3} \cdot$ \\ Giuseppe Colloca ${ }^{3}$. Giacomo Corrado ${ }^{2}$. Stefano Gentileschi ${ }^{4,5}$. Gabriella Macchia ${ }^{6}$. Enrica Tamburrini ${ }^{7}$. \\ Maria Antonietta Gambacorta ${ }^{3,8} \cdot$ Anna Fagotti $^{2,9} \cdot$ Giovanni Scambia $^{2,9} \cdot$ Gemelli Vul.Can MDT
}

Received: 16 May 2020 / Accepted: 3 July 2020 / Published online: 12 July 2020

○) Springer-Verlag GmbH Germany, part of Springer Nature 2020

\begin{abstract}
Introduction Since the community spread of Coronavirus disease 2019 (COVID-19), the practice of oncologic care at our comprehensive cancer center has changed. Postponing cancer treatment without consideration of its implications could cost more lives than can be saved. In this special situation, we must continue to provide our cancer patients with the highest quality of medical services assuring the safety. This article provides general guidance on supporting curative treatment strategies in vulvar cancer patients.

Methods At our institution, a vulvar cancer multidisciplinary team (Vul.Can MDT) of specialists is responsible for personalized treatment of this disease. The phase 2 period necessarily requires specific procedures for both outpatient and inpatient pathways and to provide strategies concerning the management of vulvar cancer patients even in case of an eventually concomitant SARS-CoV-2 infection. In brief, an accurate remote and in person triage must be provided routinely and patients submitted to specific diagnostic tests prior to every major treatment or procedure (surgery, RT, and CT) or in case of suspicion for COVID-19 syndrome. The decisional workflow for these women often old and frail, have been rapidly adjusted by our Vul.Can MDT to mitigate the potential risks of COVID-19.

Results The team produced two types of recommendations concerning: (1) safety regulations of care pathways, patients and health care providers, (2) personalized treatment strategies. We present a protocol that can be applied in clinical practice: the flowcharts provided, include the modulation of treatment intensity designed for surgical procedures and radiation, stratified for FIGO stage of disease and intention.

Conclusion We suggest that our proposals are applicable in this setting of patients, considering anyway current international recommendations and guidelines.
\end{abstract}

Keywords Vulvar neoplasms $\cdot$ COVID-19 $\cdot$ Workflow $\cdot$ Patient care $\cdot$ Treatment

\section{Background}

The outbreak of the novel Coronavirus disease 2019 (COVID-19), consisting in a severe acute respiratory syndrome often associated to multiple organ dysfunction, has rapidly spread globally, being declared a pandemic by the

Giorgia Garganese and Luca Tagliaferri contributed equally to this work and share the first authorship.

Simona Maria Fragomeni

simona.fragomeni@gmail.com

Extended author information available on the last page of the article
World Health Organization (WHO) on 11 March (Coronavirus disease 2019).

The risk to develop a severe illness by COVID-19 increases by age, comorbidities, and presence of underling medical conditions influencing immune system. Based on early statistical data, the case-fatality rate (CFR) in the patients over 60 years old is much higher than overall rate: $14.8 \%$ in patients over 80 years, $8.0 \%$ in patients aged $70-79$ years, and $3.6 \%$ in patients aged $60-69$ years (Ramella et al. 2020). The proportion of deaths over 60 years old accounts for $81 \%$ of the total deaths in the national wide, which implicates aged people are more vulnerable to the SARS-CoV-2 (Verity et al. 2020). Moreover, the overall fatality rate is $3.8 \%$ for patients with cancer, 
as a comorbid condition was $7.6 \%$ (Zhang et al. 2020; Huang et al. 2020; Aggarwal et al. 2020). In general, cancer patients must be considered more exposed and frail, because of their immunologic state, directly due to the cancer and the need for immunosuppressive anti-cancer treatments.

In particular, patients with cancer recently undergone chemotherapy and surgery seems to have more risk of COVID-19, with more severe clinical events and rapid evolution; therefore, they need to be more intensively followed (Liang et al. 2020). Some limited data on immunosuppressed cancer patients indicated a 3.5 times higher risk of needing mechanical ventilation compared with patients without cancer (Zhang et al. 2020).

Therefore, a correct management of the risk related to the circulation of SARS-CoV-2 involves many specific implications in the particular context of the geriatric oncology.

A relevant geriatric branch of gynecological oncology concerns women suffering from vulvar carcinoma, more critical because typically older and frail due to comorbidities and physical performance often aging related. Moreover, the high aggressiveness of this cancer turns every choice risky, when proceeding with treatment delivery or deciding to postpone/omit them, according to the priorities (Ramirez et al. 2020).

Gemelli University Hospital is one of the larger COVID-19 referral centre in Italy and, at the same time, one of the larger Italian Oncological Centers. In our institution, a vulvar cancer multidisciplinary team (Vul.Can MDT) is responsible for personalized treatment strategies and management. It is structured in a core team, supplemented by a group of support specialists. The core team includes two dedicated members (one senior and one young) for seven central specialties: gynecologic oncologist, plastic surgeon, radiation oncologist, medical oncologist, radiologist, nuclear medicine physician, and pathologist. The group of support specialists includes one dedicated member for each complementary specialty: geriatric oncologist, infectivologist, general surgeon, urologist, nutritionist, pain therapy anesthesiologist, psychooncologist, physiatrist, and physiotherapist. Moreover, a midwife is dedicated to the case management and a nurse provides advanced wound care. A total number of about 260 cases are annually discussed within the multidisciplinary tumor board (MDTB): about 120 are addressed to surgical procedures, among which about 30 combined with plastic surgery, 50 to radiotherapy evaluation, 15 to chemotherapy, and 10 to electrochemotherapy (ECT).

Since COVID-19 is expected to remain a primary focus of the medical leadership for a very long time, in the full absence of vulvar cancer-specific guidelines oriented to the pandemic context, we are trying to respond to the urgent need to address the possible changes of resource allocation, clinical care, and treatment delivery for vulvar cancer patients.

The aim of this paper is to focus a proposal of a personalized workflow for vulvar cancer patients and to define the specific measures that can be adopted to reduce the ongoing risk of infection during treatments.

All the contents have to be considered as an expert opinion guidance, to be considered during the extraordinary period of the pandemic emergency and do not overcome the current available international guidelines, the national and regional recommendation as well as the clinical decision obtained after interdisciplinary discussion.

\section{Methods}

The project was designed and approved in the frame of Vul. Can MDT and three steps process were defined.

In the first step, a specific Interdisciplinary Task Group, coordinated by one gynecologist (SMF) and one radiation oncologist (VL), defined preliminary postulates and guiding principles of vulvar cancer patient workflow. In the second step, the proposal was subjected to the evaluation of the Senior Members of the Vul.Can MDT and in addition, considering the issues of the project, to the infectivologist and the geriatric oncologist (GG-gynecologist, LT—radiation oncologist, SG-plastic surgeon, GCol-geriatric oncologist, GCor-medical oncologist, ET-infectivologist) who also defined management protocols, stratified for disease prognostic categories and COVID-19 status. In the third step, final evaluation was supplied by a Master Team (GS, $\mathrm{AF}, \mathrm{MAG}$, and $\mathrm{GM}$ ) for validation.

\section{Postulates and definitions}

Postulates were considered as the basic elements for the construction of renewed clinical/management pathways. Definitions were considered the way to classify elements and recognize priorities to propose targeted treatments for each category of patients (Table 1).

\section{Recommendations}

The team produced two types of recommendations: one concerning safety regulations for patient flow management and hospital environments; in particular, these have been declined in Table 2, according to three categories of relevance, referring to care pathways, patients, and health care 
providers. The second section about personalized treatment protocols in the COVID-19 era.

Since the outbreak of the pandemic, we have managed an outpatient flow of 80 patients with about 40 surgical procedures. We only recorded one case of SARS-CoV-2 infection in a patient's family member. No patient showed signs of infection due to the strict protocols of social distancing and isolation initiated even before the lockdown for the frail patients such as those typically affected by vulvar cancer.

\section{Vulvar cancer management}

Several clinical conditions have been distinguished and analyzed below, taking into account in vulvar cancer patients the frailty condition, the COVID-19 status, the stage of disease and the available treatments including surgery, radiotherapy, chemotherapy, and electrochemotherapy. The risk-benefit ratio and morbidity of these approaches were afforded in the MDTB meetings that are a requirement of utmost importance for any personalized strategy.

\section{COVID-19 symptomatic patients}

No clear guidelines exist regarding this group of patients, however, in case of acute symptomatic syndrome, the care of the COVID-19 should be reasonably considered a priority compared to anti-cancer treatments. Therefore, in patients who are candidates for diagnostics, surgical or medical treatments, it should be advisable to postpone these procedures after the complete recovery from COVID-19. Concerning palliative treatments they should be performed only when acute, severe, and extremely urgent and should be faced with the least invasive treatment: for example, in case of acute and irreversible bleeding from a bulky proliferative lesion, hemostatic radiotherapy or embolization could be provided, paying attention to carefully trace and secure the hospital staff and pathways used for the urgency. The clinical pathways for the management of women with vulva cancer who are infected with Sars-CoV-2 are shown in Fig. 1.

\section{COVID-19 asymptomatic patients}

Given the high risk of an unfavorable outcome from COVID19 , even treatments of asymptomatic or pauci-symptomatic cases, should be jointly discussed in the MDTB, strongly considering to postpone vulvar cancer cure after negative laboratory test are gained. Time interval for lab test repeat should be defined on the base of infectivologist's opinion, usually 2 weeks from the first diagnosis. In case of negativization of laboratory tests, oncological care could safely continue; in case of persistence with no symptoms, the MDTB should decide whether to proceed with the treatments limited to cases deemed urgent (e.g. aggressive disease, severe cancer-related symptoms). However, in these cases, the least invasive treatments should be carefully chosen, considering consequences related to treatment delivery or delay. Moreover, any chosen treatment should be carried out in COVID19 dedicated pathway.

\section{Switching patients}

Non-COVID patients may become positive for SARS-Cov-2 during administration of prolonged anti-cancer treatments, such as radiation therapy and chemotherapy. Continuous surveillance, repeating lab tests periodically (usually every 10-15 days), can favor early diagnosis of viral infection and prompt introduction to COVID-19 dedicated pathways, when disease is still pre- or pauci-symptomatic.

The possible decision to complete, discontinue or postpone the ongoing anti-cancer treatments requires a focused discussion in the MDTB, carefully considering risk-benefit balance, based on many contextual parameters, such as frailty, presence of COVID-19 symptoms, cancer aggressiveness and time to complete the ongoing therapies.

In case of discontinuation, treatments could potentially be resumed after recovery, whenever it is still possible and beneficial. Usually two consecutive negative laboratory tests (collected $>24 \mathrm{~h}$ apart), and a waiting time of at least 3-7 days from symptoms resolution and imaging restoration could be considered as adequate (Istituto Superiore della Sanità 2020).

In any case, we suggest to share as clearly as possible the benefits, risks, and final decisions with patient and possibly caregivers.

\section{Patients negative for COVID-19}

\section{Work up}

To minimize the exposure of patients to hospital-related risks, diagnostic procedures and patients' access should be limited at most.

The work-up should follow a fast track protocol, included at the first access:

- Integrated evaluation of the patient's general conditions with the assessment of the potential frailty

- Gynecologic exam and biopsy of the vulvar lesion, if required

- Pelvic and inguinal lymph node ultrasound, with needle-aspiration/biopsy if required 
Table 1 Postulates and definitions

Frailty

Frailty syndrome may be defined as a state of increased vulnerability resulting from the aging-associated decline in reserve and function, across multiple physiologic systems that carry an increased risk for poor health-related outcomes including mortality (Fried et al. 2001; Rockwood and Mitnitski 2007; Colloca et al. 2018). Starting from this definition, it is highlighted how it is important to define whether an oncological patient is frail or not to avoid both over and under-treatment and for a personalization of treatments (Fried et al. 2001) following the main international guidelines on cancer patients, we have distinguished the patients in "Fit" and "Frail"

In this distinction, we have also considered the factors that lead to greater death rate and have adverse effects COVID-19 related. Moreover, we considered those who are older than 60 years of age to be elderly, since from this age onwards the greatest death rate and the greatest number of respiratory complications were highlighted. The number of comorbidities, physical and cognitive performances

COVID-19 status definition

The following patients' categories are defined according to reported symptoms, imaging and lab tests results (nasopharyngeal swab/antibody tests):

Negative for COVID-19 (non-COVID): negative lab tests* (w/wo negative imaging and absence of specific symptoms)

Positive for COVID-19 (COVID): positive lab tests* and/or pathognomonic radiological imaging (w/wo specific symptoms)

Switching patient: a non-COVID patient who switch to positive during the course of treatment.

Due to patients' high risk of conversion during anti-cancer treatments, especially if extended over time — a strict surveillance is needed, provid-

ing a programmed longitudinal repetition of the triage questionnaire and lab tests

*Currently, the more widely recommended reference lab test is the nasopharyngeal swab, while many serologic tests are still under investigation; however, further rapid diagnostic tests could be available in the future (Ginocchio and McAdam 2011)

Prognostic disease categories

The following categories are defined according to the revised FIGO staging system of 2009 (Pecorelli 2009)

Early stage: stage I (small volume, node negative patients)

Locally advanced stages: stage II (diffusion to structures and organs bordering the vulva), stage III (groin lymph nodal involvement)

Extra-regional advanced stages: stage IVa/b (diffusion to pelvic organs and/or lymph node)

Metastatic disease: Stage IVb (distant organs metastasis)

Treatment intention

Type of planned treatment according to standard/international and institutional guidelines, stratified by intent

Surgery

Radical—provided both in upfront setting to obtain complete free margin excision of the disease on primary tumor site and regional lymph nodes

Debulking - surgical resection on residual disease after exclusive RT-CT; it is performed when complete response is not achieved to secure treatment effectiveness, mainly if minimal surgical effort is expected

Palliative-focused on supplying the greatest benefit using the least invasive intervention, relieving local disease-related symptoms (pain,

bleeding, poor quality of life) in patients with systemic spread, unresectable disease, or high operative risk

Radiotherapy ( \pm Chemotherapy)

Adjuvant — given in addition to the primary surgery to maximize its local effectiveness in case of risk factors

Neoadjuvant—-given before surgery to reduce the demolitive approach in extended disease or to render operable an unresectable disease

Exclusive (radical) — is given alone with radical intent on primary or recurrent disease

Palliative/temporary control—hypofractionated radiotherapy delivered to achieve a relief of local disease-related symptoms (pain, bleeding) or a delay of disease progression, even in the absence of symptoms

Chemotherapy

Neoadjuvant-before surgery to reduce tumor burden, to shrink surgical effort or to make the disease operable (mainly if radio-chemotherapy is not applicable)

Adjuvant-after radical surgery, as additional treatment only in rare cases with advanced stages of particular histotypes (i.e. invasive Paget's disease)

Palliative-aimed to favor relief from tumor-related symptoms and improve quality of life, even if a major survival advantage is not expected, provided a palliation/toxicity trade off

Radiosensitizing — associated with radiotherapy, as systemic intravenous administration that provides a cooperative effect to improve local disease control and mitigate radiation related side effects.

Targeted to the tumor site—as one-shot administration, aimed to drug transfer in tumor cells by electroporation (electrochemotherapy-ECT)

Modulation of treatment intensity (MTI)

Any modulation of treatment intensity with respect to the standard, introduced to minimize the risk of exposure to COVID-19, are coded as follows

Perform according to $\underline{\text { Standard treatment }(\mathrm{S})}$

$\underline{\text { Reduce the treatment intensity }(\mathrm{R})}$

Switch to other therapies (SW)

Postpone (P)

Omit $(\mathrm{O})$ 
Table 1 (continued)

Remote triage

It is a questionnaire which can be administered by telephone or via web, aimed at establishing the risk of contagion of the interviewee, in the time interval of the last two weeks, by asking questions

Epidemiologically oriented, to investigate whether she had contacts, occasions or experiences at risk of contagion with SARS-CoV-2

Clinically oriented, to determine if she experienced clinical symptoms attributable to COVID-19

Hospital triage

Patients with negative remote triage should be admitted to hospital triage (in person)

Before accessing the outpatient service or the hospitalization, they should be subjected to

a. Repetition of the questionnaire administered by remote triage

b. Body temperature detection

c. Lab test: always prior to hospitalization or in case of suspicion of SARS-CoV2 contagion

In case of a negative hospital triage patient, should be admitted; in case of positivity, patient should be addressed to the COVID-19 pathway

- Blood-chemical and serological investigations (including SCC tumor marker)

On the basis of this fast track protocol, the gynecologist should preliminarily assess the clinical stage and follow these advices:

Clinical early stage: if lymph nodes are negative at ultrasound assessment, given the accuracy of the ultrasound exam when performed by skilled examiner, considering the low risk for distant metastasis, we could suggest to omit additional imaging to complete systemic staging (Frumovitz et al. 2008; Gradishar et al. 2020).

Clinical advanced stage: if groin or pelvic lymph nodes show metastatic involvement at ultrasound or if infiltration of cutaneous bridges, urethra, anus, or vagina are evident or suspicious, considering the higher risk for distant metastases, a whole-body assessment should be performed by conventional radiologic imaging (CT, plus or minus pelvic MRI) or metabolic imaging (by ${ }^{18 \mathrm{~F}} \mathrm{FDG}-\mathrm{PET} / \mathrm{CT}$ ) (Salani et al. 2017; Kataoka et al. 2010; Robertson et al. 2016; Viswanathan et al. 2013; Collarino et al. 2018, 2017; Fiorentino et al. 2019; Alongi et al. 2019).

In case of undefined clinical presentation, a case-bycase preoperative work-up should be planed after MDTB discussion.

\section{Treatment}

Several clinical conditions have been distinguished and analysed below, taking into account all potential treatments among the surgical, radiotherapy, and chemotherapy options.

\section{Fit patients}

Radical surgery on primary tumor site and groin lymph nodes should be highly supported but keeping in mind that impact of surgical burden should be limited as much as possible. If plastic surgery is required to repair tissue defects, techniques producing minimal impact should be favored
(Gentileschi et al. 2016, 2017). Medical therapies (RT and CT) should be guaranteed, anyway favoring the least possible impact. In particular, neoadjuvant treatment has not been included among the available treatment options because of the high cumulative morbidity of RT-CT followed by radical surgery, in the absence of a strong evidence of benefit compared to exclusive RT regimen (Montana et al. 2000; Moore et al. 2012).

All these general principles fit for all stages. Possible modulations on the standard therapeutic choices are reported in Tables 3 and 4.

Early stages Standard radical surgery with negative surgical margins should be performed on primary tumor site if possible. Sentinel lymph node procedure has to be always provided in all clinically N0 patients that meet the standard criteria (primary unifocal tumor $<4 \mathrm{~cm}$ ) (Frumovitz et al. 2008; Gradishar et al. 2020). The remaining clinically N0 cases, not fit for the standard selection criteria, should be addressed to radical lymphadenectomy, with high rate of morbidity and complications, often unnecessary. Nevertheless, considering the need for reducing the severe morbidity related to this surgical procedure (DiSaia et al. 1979; Carlson et al. 2018), a sentinel lymph node biopsy could be considered instead of standard radical lymphadenectomy always after discussion in MDTB. Recent preliminary evidences showed the safety of the procedure even in this setting only if provided that an accurate ultrasound and metabolic (PET/CT) assessment of the lymph node status is performed by expert examiners (Garganese et al. 2017, 2020). Possible modulation in radiation therapy approach is reported in Table 4.

Locally advanced stages The standard treatment may include exclusive radio-chemotherapy (RT-CT) or radical surgery eventually followed by adjuvant RT.

Radical lymphadenectomy should be performed in all cases with proven or highly suspicious inguinal lymph node 
Table 2 Guiding principles

\section{Care pathways}

Provide for a clear separation of the care pathways addressed to non-COVID and COVID patients with vulvar cancer, organized in different spaces, days or times of access

Combine care services provided remotely (by telemedicine) with those performed in person (at home and hospital), limiting as much as possible the patients' need for hospital access

Standardize staff training for telemedicine and home support services

Perform in advance remote triage to each patient who is scheduled for hospital access (both on an outpatient and hospitalization) (see Table 1)

Repeat a hospital triage to all patients entering the hospital (see Table 1)

Submit patients to diagnostic lab test prior to every major treatment or procedure (surgery, RT, and CT) and provide a longitudinal continuous

bio-surveillance during and after treatments (see Table 1)

Provide for laboratory diagnostic tests as reliable and rapid as possible

Combine the largest number of activities deliverable during an outpatient visits or hospitalization, to minimize the number of patients' admissions (e.g. combine first gynecological examination with inguinal lymph nodes ultrasound, biopsy of suspicious lesions and pain management consultancy)

Avoid as much as possible the use of waiting rooms: in any case provide for spacious settings, measures for interpersonal safety distance, adequate airing of the environment and supply with hand hygiene devices

Limit the hospital access to visitors and accompanist, excepting for patients with severe disabilities and limited to the occasions in which assistance is not provided by hospital staff

Activate protocols for the adequate and repeated sanitation, keeping safe environment to work and care for patients

Discuss in a multidisciplinary board the precise planning for the optimal treatment course of any patient, to limit the number of elective procedures

Provide web platform to perform multidisciplinary tele-conferences, to improve collaboration with distant health care providers that needs to refer to an expert dedicated team

Provide specific indications about other proper healthcare facilities where to address patients to receive prolonged treatment, according to the type of therapy proposed and patient's residence

\section{Staff}

Limit the number of on-site staff members during frontal activities with patients

Standardize protocols for the reduction of the risk of contagion, even in the non-COVID pathways, providing the use of most adequate personal protective equipment (PPE) and the interpersonal safety distance in the visit rooms (except for moments of clinical exam or diagnostic procedures)

Minimize the time for clinical visits providing indirect or telematic supply for preliminary/informative procedures not requiring "in person" activities, such as clinical history and instrumental exams collection, some informative communications and consent procedures

Assign a well selected medical staff to the services in which multitasking skills are required, to deliver the highest possible number of services at any single access of patients to the hospital: for example, gynecologists capable of performing the first dedicated oncological visit together with a diagnostic ultrasound examination and/or a vulvar or ultrasound-guided lymph node biopsy

Split healthcare personnel into stable small work teams (to limit the effects resulting from a possible contagion of a team member to a small group only)

Adopt protocols for continuous bio-surveillance of healthcare staff, repeating diagnostic laboratory tests for SARS-CoV-2 infection. Our proposal is: every 10-15 days, until other risk control measures are available

\section{Patients}

Advice patients to use PPE to enter the hospital; for those unprovided, supply them before entering services

Carefully evaluate patients' frailty (basing on factors such as age, comorbidities and performance) related to the risk of a possible severe clinical evolution from COVID-19

Advise patients to limit their exposure only to family members or cohabiters, since they are recognized as a high-risk population.

Ask the relatives of surgical candidates to donate blood

Adopt adequate bio-surveillance protocols for patients during anti-cancer treatments, repeating diagnostic lab tests for SARS-CoV-2 infection.

Our proposal is

24-48 $\mathrm{h}$ before surgery and then every $10-15$ days during the postoperative period, until 30 days after the healing of surgical wounds $24-48 \mathrm{~h}$ before the start of radiotherapy or chemotherapy, then approximately every 10-15 days during treatment and up to 30 days after the end of the treatments

Ensure communication between patients and their relatives/caregivers by phone or video-call during hospitalization for anti-cancer treatments Supply integrative supportive care during hospitalization

Ensure continuous and comprehensive remote supplementary home care during anti-cancer treatments, privileging video visits or telephone encounters for psycho-oncological support (intended for patients and their relatives/care givers), nutritional consultancy, palliative care and all the other integrative therapies

metastasis, taking care to completely harvest the superficial and deep lymph nodes of the inguino-femoral triangle.

Conversely, sentinel node biopsy of lymph node surgical staging could also be considered in selected clinically
N0 cases, such as in FIGO stage III contralaterally to a metastatic groin or in FIGO stage II, beyond the standard selection criteria for SLN, limited to the conditions previously specified in early stage workflow. Moreover, complete 


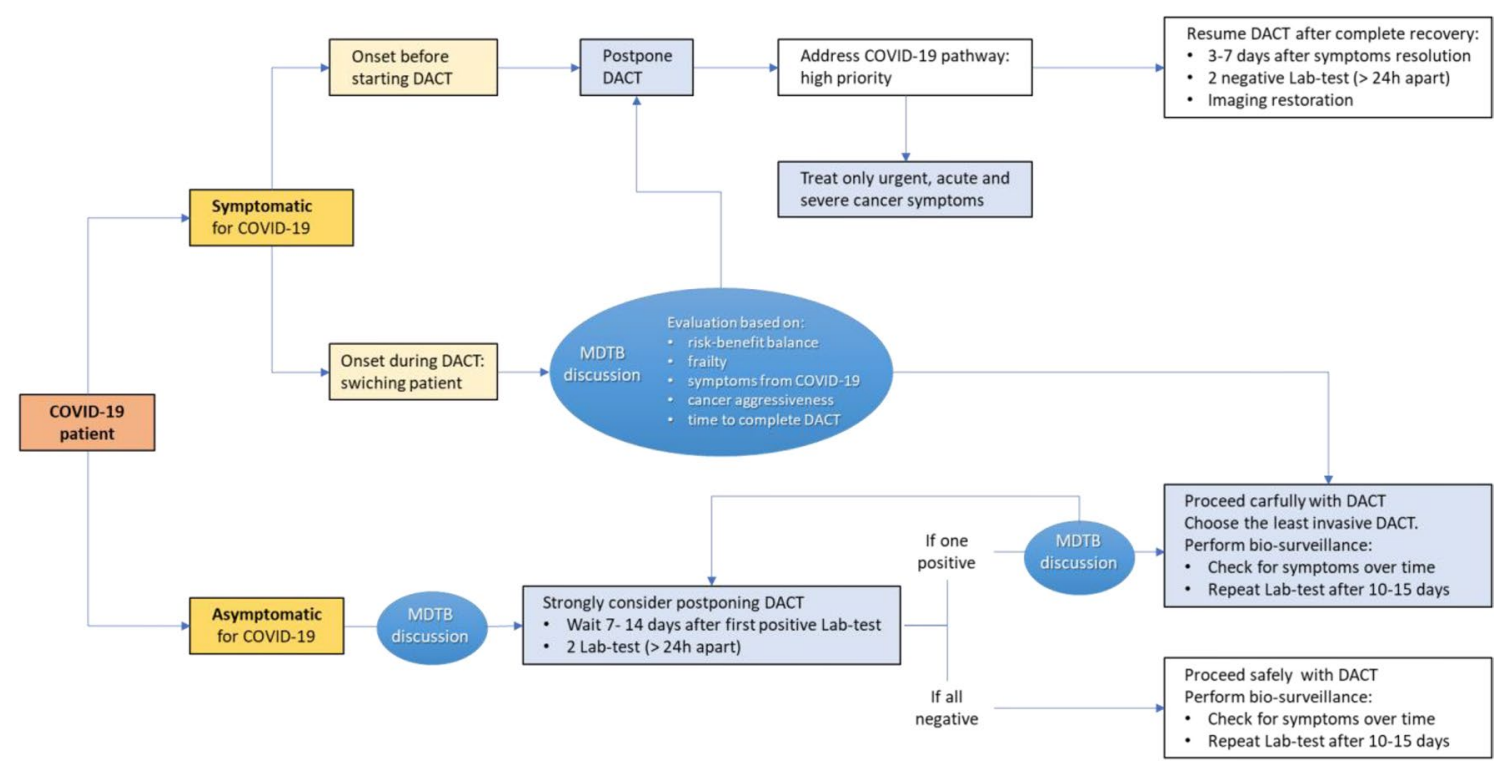

Fig. 1 Clinical pathways for management of patients with SARS-CoV2 infection and vulvar cancer. DACT diagnostic tests and anti-cancer treatments

omission of surgical staging could be considered after MDTB.

In case of perineal extensive disease, requiring large surgical demolition of structures bordering the vulva (urethra, vagina or anus), with possible functional impairment, and/ or large plastic reconstruction, upfront radical RT should be considered, both in node negative or positive patients, after MDTB discussion. In this case, RT should be delivered with radical intent and surgery would be performed only on residual disease with debulking intent.

Local recurrence The choice underlies the treatments previously carried out and the extent of disease. Surgery could still obtain radical results; radical RT-CT could be the favorite option in large relapses followed by debulking surgery in case of partial response. ECT or other palliative treatments for local control of disease (surgery or RT regimens) could take over if the radical intent can no longer be pursued (Certelli et al. 2020).

Metastastatic stage Chemotherapy with or without palliative local treatments versus supportive palliative care programs are suggested. The MDTB choice should focus on possible benefits of proposed treatments (Weinberg and Gomez-Martinez 2019).

\section{Frail patients}

In this setting, the MDTB discussion is particularly required to define the most balanced and personalized treatment plan.
Early stage Radical surgery has to be considered limited to cases requiring low surgical effort. In larger lesions, one option could be upfront palliative surgery, with minimally invasive approach on primary tumor site, even omitting sentinel node procedure in previous well staged $\mathrm{cN} 0$ cases. Another option could be the shift to other locoregional palliative treatments (e.g. RT regimens or ECT).

Locally advanced stages The most recommended choice might be the primary RT-CT treatment with exclusive intent. In case of minimal residual disease debulking surgery could still play a role. In large volume residual disease, we suggest to consider to omit surgery, evaluating switch to alternatives such as chemotherapy, interventional miniinvasive local therapy (brachitherapy, stereotactic RT, ECT) or palliative care.

Moreover, palliative primary tumor site surgery and/or selective lymphadenectomy could be considered.

Local recurrences and metastastic stages Decisions regarding initiation of additional chemotherapy or further local treatments should be based on clinical MDTB judgment and potential for benefit based on expected response of subsequent available therapies.

\section{Follow-up}

Any patient home management should be encouraged, including telemedicine and phone calls. It is well kwon than only visual and clinical inspection permits early detection of 
Table 3 Flowchart of surgical indications, stratified for clinical presentation

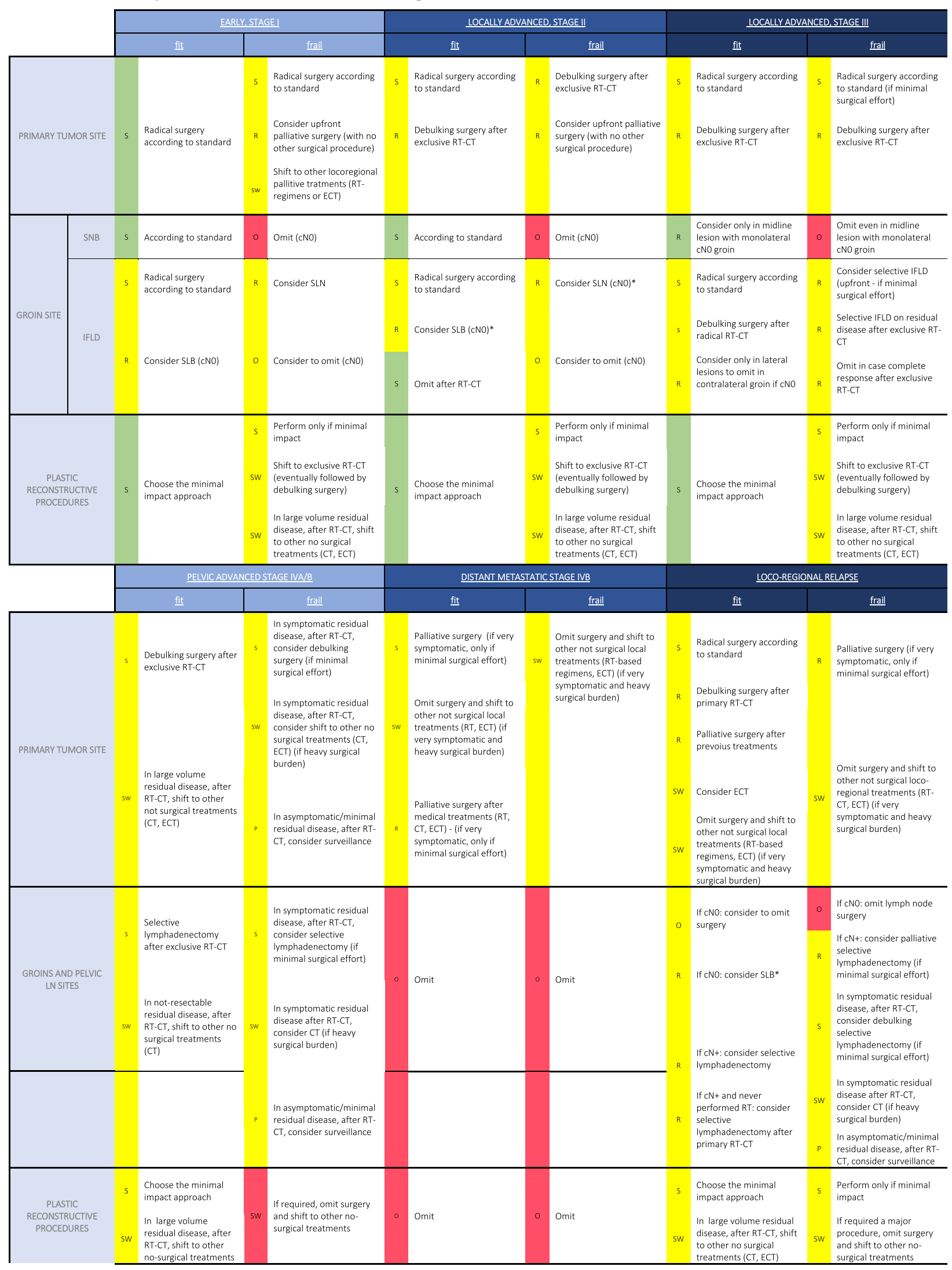

Color code: a) green is for one single viable option; b) yellow is for possibility to choose between multiple available options (MDTB discussion is required); c) red is for one single option that is to omit the treatment. SNB sentinel lymph node biopsy; IFLD radical inguino-femoral lym- 
Table 3 (continued)

phadenectomy; $R T$ radiotherapy; $C T$ chemotherapy; $E C T$ electrochemotherapy; $S$ perform according to $\underline{S}$ tandard treatment; $R \underline{R} \underline{\text { Reduce the treat- }}$

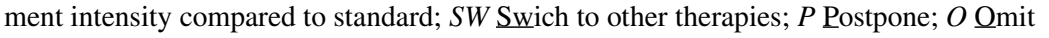

Table 4 Flowchart of indications to radiotherapy stratified for intention

\begin{tabular}{|c|c|c|c|c|c|c|}
\hline \multirow[b]{2}{*}{ ADJUVANT } & \multirow{2}{*}{\multicolumn{2}{|c|}{$\frac{\text { PRE-COVID-19 ERA }}{\text { ALL }}$}} & \multicolumn{4}{|c|}{ COVID-19 ERA } \\
\hline & & & \multicolumn{2}{|r|}{$\overline{\mathrm{FHT}}$} & \multicolumn{2}{|r|}{$\overline{\text { FRAIL }}$} \\
\hline $\begin{array}{l}\text { Major criteria for indication } \\
>\text { at least } 1 \text { for indication }\end{array}$ & $\mathrm{s}$ & $\begin{array}{l}\text { Margins status } \\
\text { Close: }<8 \mathrm{~mm} \text { at the definitive histology } \\
\text { (if a second surgery is not } \\
\text { planned/possible) } \\
\text { Positive (R1): presence of focal } \\
\text { microscopic disease (if a second surgery } \\
\text { is no planned/possible) } \\
\text { Tumor depth of invasion }>5 \mathrm{~mm} \\
\text { Nodal status: single positive lymph node if } \\
\text { metastasis diameter is } \leq 2 \mathrm{~mm}\end{array}$ & $\mathrm{R}$ & $\begin{array}{l}\text { Margins status: } \\
\text { Positive (R1): presence of focal } \\
\text { microscopic disease if a second surgery } \\
\text { is not planned/possible) } \\
\text { Nodal status: } \\
\text { One single positive lymph node if } \\
\text { metastasis diameter is }>2 \mathrm{~mm} \text { (discuss in } \\
\text { MDTB the opportunity to concomitant } \\
\text { chemotherapy) }\end{array}$ & $\mathrm{R}$ & $\begin{array}{l}\text { Margins status: } \\
\text { Positive (R1): presence of focal } \\
\text { microscopic disease (if a second surgery is } \\
\text { not planned/possible } \\
\text { Positive (R2) due macroscopic disease (if } \\
\text { a second surgery is not planned/possible) } \\
\text { Nodal status: } \\
\text { single positive lymph node if metastasis } \\
\text { diameter is }>2 \mathrm{~mm}\end{array}$ \\
\hline $\begin{array}{l}\text { Intermediate criteria for indication } \\
\\
\text { to submit at MDTB discussion: final } \\
\text { decision will be defined according to } \\
\text { frailty assessment }\end{array}$ & & & $\mathrm{R}$ & $\begin{array}{l}\text { O Margins status: } \\
\text { Close: }<8 \mathrm{~mm} \text { at the definitive histology } \\
\text { (if a second surgery is not } \\
\text { planned/possible) } \\
\text { Tumor depth of invasion }>5 \mathrm{~mm} \\
\text { Nodal status: } \\
\text { One single positive lymph node if } \\
\text { metastasis diameter is }<2 \mathrm{~mm} \text { (if radical } \\
\text { inguinal surgery has been completed) }\end{array}$ & & $\begin{array}{l}\text { Nodal status: single positive lymph node if } \\
\text { metastasis diameter is }<2 \mathrm{~mm} \text { (if radical } \\
\text { inguinal surgery has been completed) }\end{array}$ \\
\hline $\begin{array}{l}\text { Minor criteria for indication } \\
\text { > not sufficient, a priori, to prescribe } \\
\text { adjuvant radiotherapy, but useful } \\
\text { elements for discussion in the MDTB. } \\
\text { The final decision in some cases could } \\
\text { be made considering also these minor } \\
\text { criteria and the patient's general } \\
\text { conditions }\end{array}$ & $\mathrm{s}$ & $\begin{array}{l}\text { - Lymphovascular invasion } \\
\text { - Tumor size }>4 \mathrm{~cm} \\
\text { - Multifocal tumor } \\
\text { - Grading G3 } \\
\text { - Anterior tumor site } \\
\text { - Loco-regional } \\
\text { - Recurrence after previous surgery }\end{array}$ & $\mathrm{R}$ & $\begin{array}{l}\text { - Lymphovascular invasion } \\
\text { - Tumor size }>4 \mathrm{~cm} \\
\text { - Multifocal tumor } \\
\text { - Grading G3 } \\
\text { - Anterior tumor site } \\
\text { - Loco-regional } \\
\text { - Recurrence after previous surgery }\end{array}$ & $\mathrm{R}$ & $\begin{array}{l}\text { - Lymphovascular invasion } \\
\text { - Tumor size }>4 \mathrm{~cm} \\
\text { - Multifocal tumor } \\
\text { O Grading G3 } \\
\text { - Anterior tumor site } \\
\text { - Loco-regional } \\
\text { - Recurrence after previous surgery } \\
\text {. Margins status: } \\
\text {. Close: }<8 \text { mm at the definitive histology } \\
\text { (if a second surgery is not } \\
\text { planned/possible) } \\
\circ \text { Tumor depth of invasion }>5 \mathrm{~mm}\end{array}$ \\
\hline $\begin{array}{l}\frac{\text { Criteria for concomitant }}{\text { chemotherapy }} \\
>\text { at least } 1 \text { for indication }\end{array}$ & s & $\begin{array}{l}\text { - Margins status: } \\
\text { - Positive (R2) due macroscopic disease (if } \\
\text { a second surgery is not planned/possible) } \\
\\
\text { Nodal status: } \\
\text { One single positive lymph node if } \\
\text { metastasis diameter is }<2 \mathrm{~mm} \text { (if radical } \\
\text { inguinal surgery has been completed) } \\
\text { - Presence of } 2 \text { or more positive lymph } \\
\text { nodes Presence of node with ECE }\end{array}$ & $\mathrm{R}$ & $\begin{array}{l}\text { Margins status: } \\
\text { - Positive }(\mathrm{R} 2 \text { ) due macroscopic disease } \\
\text { (if a second surgery is not } \\
\text { planned/possible) } \\
\text { Nodal status: } \\
\text { - Presence of } 2 \text { or more positive lymph } \\
\text { nodes } \\
\text { - Presence of node with ECE }\end{array}$ & $\mathrm{R}$ & $\begin{array}{l}\text { - Nodal status: } \\
\text { - Presence of node with ECE (discussing in } \\
\text { MDTB the opportunity }\end{array}$ \\
\hline \multirow[t]{2}{*}{ NEOADJUVANT } & & $\overline{\text { ALL }}$ & \multicolumn{2}{|c|}{ 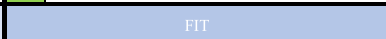 } & \multicolumn{2}{|r|}{ FRAIL } \\
\hline & $\mathrm{s}$ & $\begin{array}{l}\text { to reduce the demolitive approach in extended } \\
\text { disease or to convert an unresectable disease to } \\
\text { operable }\end{array}$ & $\stackrel{\mathrm{s}}{\mathrm{w}}$ & switch to exclusive $\mathrm{RT} / \mathrm{CT}$ & $\begin{array}{l}\mathrm{S} \\
\mathrm{W}\end{array}$ & switch to palliative/ temporary control \\
\hline EXCLUSIVE (RADICAL) & & ALL & \multicolumn{2}{|r|}{$\overline{F H I}$} & \multicolumn{2}{|r|}{$\overline{\text { FRAIL }}$} \\
\hline $\begin{array}{l}\text { Radiotherapy and concomitant } \\
\text { chemotherapy (RT/CT) }\end{array}$ & s & $\begin{array}{l}\text { RT/CT: Local Advanced with or without nodal } \\
\text { involvement }\end{array}$ & $\mathrm{s}$ & $\begin{array}{l}\text { RT/CT: Local Advanced with or without nodal } \\
\text { involvement } \\
\text { Consider Simultaneous Integrated boost and } \\
\text { Interventional Radiotherapy boost in order to } \\
\text { reduce the radiotherapy fraction }\end{array}$ & $\begin{array}{l}\mathrm{S} \\
\mathrm{W}\end{array}$ & omit and switch to palliative/ temporary control \\
\hline \multirow[t]{2}{*}{$\begin{array}{l}\text { PALLIATIVE / TEMPORARY } \\
\text { CONTROL }\end{array}$} & & ALL & & FII & \multicolumn{2}{|r|}{ FRAIL } \\
\hline & $\mathrm{s}$ & $\begin{array}{l}\text { Long course (e.g. 3Gy x } 10 \mathrm{Frx} \text { ) } \\
\text { Short course: (e.g 4Gy x } 4-5 \mathrm{Frx} \text { ) (to prefer in } \\
\text { case of symptoms) }\end{array}$ & $\underline{\mathrm{S}}$ & $\begin{array}{l}\text { Long course (e.g. 3Gy x } 10 \mathrm{Frx} \text { ) } \\
\text { Short course: (e.g 4Gy x } 4-5 \mathrm{Frx} \text { ) (to prefer in } \\
\text { case of symptoms) }\end{array}$ & $\mathrm{R}$ & Short course: (e.g 4Gy x 4-5 Frx) \\
\hline
\end{tabular}

Color code: a) green is for one single viable option; b) yellow is for possibility to choose between multiple available options (MDTB discussion is required); c) red is for one single option that is to omit the treatment. $R 1$ microscopic residual disease on surgical margins; $R 2$ macroscopic residual disease on surgical margins; $R T$ radiotherapy; $C T$ chemotherapy; $S$ perform according to Standard treatment; $R$ Reduce the treatment

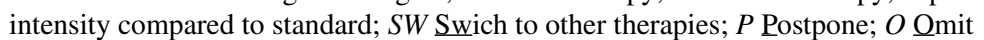


recurrence, preventing an incurable progression: thus, as a compromise, we suggest every 4 months follow-up evaluations alternating telemedicine and outpatient visits. Virtual consultation should provide questionnaires including specific items aimed to identify the early symptoms and signs of a possible recurrence. It would be very useful to ensure the presence of the care giver next to the patient, to collect advice from a person able to perform an elementary anatomical inspection and to offer additional information about the symptoms and disorders reported.

Results from each virtual consultation should be recorded and if any doubt arises about a possible relapse, a medical exam should be scheduled in a short time.

\section{Conclusions}

In this manuscript, we have summarized our internal guidelines to manage vulvar cancer in COVID-19 era.

Our model is focused on finding a compromise between the risks of SARS-CoV-2 infection and the need to ensure the best oncologic treatments.

In this scenario, the application of the current available guidelines still remains a priority.

Each deviation from standard or modulation of treatment options for particular conditions requires a critical approach, individually customized, within the frame of a MDTB discussion.

The experience of the referral centers with dedicated MDTBs needs to be shared and extended to other centers through the use of multimedia support.

This expert-opinion-based guideline could be considered as a resource in an unprecedented critical period, still evolving. Moreover, the suggestions provided have to be molded on national circumstances and existing health system regulations.

Acknowledgements Garganese Giorgia-Gynecologic oncologist (Gynecology and Breast Care Center, Mater Olbia Hospital, Olbia, Italy; UOC Ginecologia Oncologica, Dipartimento Scienze della Salute della Donna, del Bambino e di Sanità Pubblica, Fondazione Policlinico Universitario A. Gemelli IRCCS, Rome, Italy), Simona Maria Fragomeni-Gynecologic oncologist (UOC Ginecologia Oncologica, Dipartimento Scienze della Salute della Donna, del Bambino e di Sanità Pubblica, Fondazione Policlinico Universitario A. Gemelli IRCCS, Rome, Italy), Tagliaferri Luca-Radiation oncologist (UOC di Radioterapia Oncologica, Dipartimento Diagnostica per Immagini, Radioterapia Oncologica e Ematologia, Fondazione Policlinico Universitario A. Gemelli IRCCS, Rome, Italy), Valentina Lancellotta-Radiation oncologist (UOC di Radioterapia Oncologica, Dipartimento Diagnostica per Immagini, Radioterapia Oncologica e Ematologia, Fondazione Policlinico Universitario A. Gemelli IRCCS, Rome, Italy), Vittoria Rufini-nuclear medicine physician, Luca Zagaria-nuclear medicine physician, Angela Collarino-Nuclear medicine physician, Frediano Inzani-Pathologist, Zannoni Gian Franco-Pathologist, Giacomo Corrado-Medical oncologist (UOC Ginecologia Oncologica,
Dipartimento Scienze della Salute della Donna, del Bambino e di Sanità Pubblica, Fondazione Policlinico Universitario A. Gemelli IRCCS, Rome, Italy), Ferrandina Gabriella-Medical Oncologist, Stefano Gentileschi-Plastic surgery (UOC Chirurgia Plastica, Dipartimento Scienze della Salute della Donna, del Bambino e di Sanità Pubblica, Fondazione Policlinico Universitario A. Gemelli IRCCS, Rome, Italy; Istituto di Clinica Chirurgica, Università Cattolica del Sacro Cuore, Rome, Italy), Gianluigi Stefanizzi-Plastic surgery, Benedetta GuiRadiologist, Salvatore Persiani-Radiologist.

Funding None.

\section{Compliance with ethical standards}

Conflict of interest The authors declare that they have no conflict of interest.

\section{References}

Aggarwal S, Garcia-Telles N, Aggarwal G, Lavie C, Lippi G, Henry BM (2020) Clinical features, laboratory characteristics, and outcomes of patients hospitalized with coronavirus disease 2019 (COVID-19): early report from the United States. Diagnosis (Berl) 7:91-96

Alongi P, Laudicella R, Desideri I et al (2019) Positron emission tomography with computed tomography imaging (PET/CT) for the radiotherapy planning definition of the biological target volume: Part 1. Crit Rev Oncol Hematol 140:74-79

Carlson JW, Kauderer J, Hutson A et al (2018) GOG 244, the lymphedema and gynecologic cancer (LEG) study: incidence and risk factors in newly diagnosed patients. Gynecol Oncol 149:6-7

Certelli C, Garganese G, Fragomeni SM et al (2020) Electrochemotherapy in vulvar cancer: a systematic review It. J Obstet Gynaecol 32:34-43

Collarino A, Garganese G, Valdés Olmos RA et al (2017) Evaluation of dual-timepoint 18F-FDG PET/CT imaging for lymph node staging in vulvar cancer. J Nucl Med 58:1913-1918

Collarino A, Garganese G, Fragomeni SM, et al (2018) Radiomics in vulvar cancer: first clinical experience using 18F-FDG PET/CT images. J Nucl Med. pii: jnumed.118.215889.

Colloca G, Di Capua B, Bellieni A et al (2018) Muscoloskeletal aging, sarcopenia and cancer. J Geriatr Oncol 10:839

Coronavirus disease 2019 (COVID-19) Situation report-72 highlights. www.who.int/epi-win. Accessed 6 Apr 2020.

DiSaia PJ, Creasman WT, Rich WM (1979) An alternate approach to early cancer of the vulva. Am J Obstet Gynecol 133:825-832

Fiorentino A, Laudicella R, Ciurlia E et al (2019) Positron emission tomography with computed tomography imaging (PET/CT) for the radiotherapy planning definition of the biological target volume: Part 2. Crit Rev Oncol Hematol 139:117-124

Fried LP, Tangen CM, Walston J et al (2001) Frailty in older adults: evidence for a phenotype. J Gerontol A Biol Sci Med Sci 56:M146-M156

Frumovitz M, Charles F, Levenback CF (2008) Lymphatic mapping and sentinel node biopsy in vulvar, vaginal, and cervical cancers. Oncology (Williston Park) 22:529-536

Garganese G, Collarino A, Fragomeni SM et al (2017) Groin sentinel node biopsy and 18F-FDG PET/CT-supported preoperative lymph node assessment in cN0 patients with vulvar cancer currently unfit for minimally invasive inguinal surgery: The GroSNaPET study. Eur J Surg Oncol 43:1776-1783

Garganese G, Fragomeni SM, Pasciuto T et al (2020) Ultrasound morphometric and cytologic preoperative assessment of inguinal 
lymph-node status in women with vulvar cancer: MorphoNode study. Ultrasound Obstet Gynecol 55:401-410

Gentileschi S, Servillo M, Garganese G et al (2016) Surgical therapy of vulvar cancer: how to choose the correct reconstruction? J Gynecol Oncol 27:e60

Gentileschi S, Servillo M, Garganese G et al (2017) Versatility of pedicled anterolateral thigh flap in gynecologic reconstruction after vulvar cancer extirpative surgery. Microsurgery 37:516-524

Ginocchio CC, McAdam AJ (2011) Current best practices for respiratory virus testing. J Clin Microbiol 49(9):S44-S48

Huang Z, Zhuang D, Xiong B, Deng DX, Li H, Lai W (2020) Occupational exposure to SARS-CoV-2 in burns treatment during the COVID-19 epidemic: specific diagnosis and treatment protocol. Biomed Pharmacother 127:110176

Istituto Superiore della Sanità, Rapporto ISS COVID-19, n. 1 e 2/2020, https://www.iss.it/rapporti-covid-19.

Kataoka MY, Sala E, Baldwin P et al (2010) The accuracy of magnetic resonance imaging in staging of vulvar cancer: a retrospective multi-centre study. Gynecol Oncol 117:82-87

Liang W, GuaN W, Chenb R et al (2020) Cancer patients in SARSCoV-2 infection: a nationwide analysis in China. Lancet Oncol 21:335-337

Montana GS, Thomas GM, Moore DH et al (2000) Preoperative chemo-radiation for carcinoma of the vulva with N2/N3 nodes: a gynecologic oncology group study. Int J Radiat Oncol Biol Phys 48:1007-1013

Moore DH, Ali S, Koh WJ et al (2012) A phase II trial of radiation therapy and weekly cisplatin chemotherapy for the treatment of locally-advanced squamous cell carcinoma of the vulva: a gynecologic oncology group study. Gynecol Oncol 124:529-533

NCCN Guidelines: Gradishar WJ, Anderson BO, Balassanian R, et al. NCCN clinical practice guidelines in oncology: Vulvar cancer. Version 3.2014. Available at: NCCN.org. Accessed 19 Apr 2020.

Pecorelli S (2009) Revised FIGO staging for carcinoma of the vulva, cervix, and endometrium. Int J Gynaecol Obstet 105:103-104
Ramella S, Floreno B, D’Angelillo RM, Campanozzi LL, Tambone V (2020) Can we prevent COVID-19 from causing victims among uninfected cancer patients? Radiother Oncol. pii: S0167-8140(20)30209-7.

Ramirez PT, Chiva L, Eriksson AGZ et al (2020) COVID-19 global pandemic oncology: options for management of gynecologic cancers. Int J Gynecol Cancer 30:561-563

Robertson NL, Hricak H, Sonoda Y et al (2016) The impact of FDG$\mathrm{PET} / \mathrm{CT}$ in the management of patients with vulvar and vaginal cancer. Gynec Oncol 140:420-424

Rockwood K, Mitnitski A (2007) Frailty in relation to the accumulation of deficits. J Gerontol A Biol Sci Med Sci 62:722-727

Salani R, Khanna N, Frimer M et al (2017) An update on post-treatment surveillance and diagnosis of recurrence in women with gynecologic malignancies: Society of 2 Gynecologic Oncology (SGO) recommendations. Gynecol Oncol 146:3-10

Verity R, Okell LC, Dorigatti I, et al (2020) Articles estimates of the severity of coronavirus disease 2019: a model-based analysis. Lancet Infect Dis. pii: S1473-3099(20)30243-7.

Viswanathan C, Kirschner K, Truong M et al (2013) Multimodlity imaging of vulvar cancer: staging, therapeutic response, and complications. AJR AM J Roentgenol 200:1387-1400

Weinberg D, Gomez-Martinez RA (2019) Vulvar cancer. Obstet Gynecol Clin North Am 46:125-135

Zhang J, Yu M, Tong S, Liu LY, Tang LV (2020) Predictive factors for disease progression in hospitalized patients with coronavirus disease 2019 in Wuhan, China. J Clin Virol 28(127): 104392

Publisher's Note Springer Nature remains neutral with regard to jurisdictional claims in published maps and institutional affiliations.

\section{Affiliations}

\section{Giorgia Garganese $^{1,2} \cdot$ Luca Tagliaferri $^{3} \cdot$ Simona Maria Fragomeni ${ }^{2}\left(\right.$ Lalentina Lancellotta $^{3}$. Giuseppe Colloca ${ }^{3}$ - Giacomo Corrado ${ }^{2}$. Stefano Gentileschi ${ }^{4,5}$. Gabriella Macchia ${ }^{6}$. Enrica Tamburrini ${ }^{7}$. Maria Antonietta Gambacorta ${ }^{3,8} \cdot$ Anna Fagotti $^{2,9} \cdot$ Giovanni Scambia $^{2,9} \cdot$ Gemelli Vul.Can MDT}

1 Gynecology and Breast Care Center, Mater Olbia Hospital, Olbia, Italy

2 UOC Ginecologia Oncologica, Dipartimento Scienze della Salute della Donna, del Bambino e di Sanità Pubblica, Fondazione Policlinico Universitario A. Gemelli IRCCS, Largo A. Gemelli 8, 00168 Rome, Italy

3 UOC di Radioterapia Oncologica, Dipartimento Diagnostica per Immagini, Radioterapia Oncologica e Ematologia, Fondazione Policlinico Universitario A. Gemelli IRCCS, Rome, Italy

4 UOC Chirurgia Plastica, Dipartimento Scienze della Salute della Donna, del Bambino e di Sanità Pubblica, Fondazione Policlinico Universitario A. Gemelli IRCCS, Rome, Italy
5 Istituto di Clinica Chirurgica, Università Cattolica del Sacro Cuore, Rome, Italy

6 Radiotherapy Unit, Gemelli Molise Hospital, Università Cattolica del Sacro Cuore, Campobasso, Italy

7 UOC Malattie Infettive, Dipartimento Scienze di Laboratorio e Infettivologiche, Fondazione Policlinico Universitario A. Gemelli IRCCS, Rome, Italy

8 Istituto di Radiologia, Università Cattolica del Sacro Cuore, Rome, Italy

9 Istituto di Clinica Ostetrica e Ginecologica, Università Cattolica del Sacro Cuore, Rome, Italy 